\title{
Research on Dynamic Combination Technique Based on Capability Object in Simulation Application
}

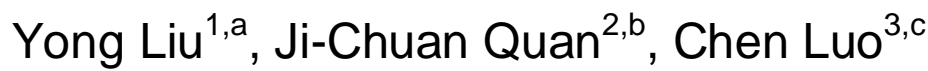 \\ 1,2,3 Institute of Command Information System,PLA University of Science and Technology, NanJing \\ 210007, China \\ aliuyong4425@sina.com, ${ }^{\mathrm{b}}$ qjch_cn@sina.com, ${ }^{\mathrm{C} I c 0810 @ 126 . c o m}$
}

Keywords: Capability model, Dynamic combination, Automated planning.

\begin{abstract}
As a new simulation operation mode, task oriented simulation can build simulation application quickly to assess the influence of commander's decision on future battlefield situation. However, with the war of information era changing dynamically and becoming unforeseeable, higher requirements for the efficiency of simulating dynamic combination and the scale are needed to meet. So based on the object task analysis approach of capability oriented, this paper first describes the simulation dynamic combination analysis process based on capability model. Then combined with Case-Based Reasoning (CBR) and Hierarchical Task Network (HTN), proposes a simulation combination algorithm based on CBR and HTN, which can solve complex questions well and improve the capability of simulation application dynamic combination. Lastly, an case is given to verify and analyze the algorithm.
\end{abstract}

\section{Introduction}

Due to the complexity of information warfare, it's difficult to predict the progress. And it needs to make real-time decision and adjust the plan all the time during the process of battle. By the use of simulation system, the deduction and planning of operational plan can do help to real-time decision support. Meanwhile, task-oriented simulation, as a new and different simulation operation mode, can build quickly and reflect the implication of each decision about future battlefield situation each time. That is the research focus in the military modeling and simulation field, and also a difficult question that hasn't been solved completely. Therefore, commanders hope to provide some more effective planning algorithms, such as CBR, HTN, Multi-agent Planning (MAP), Ontology-Based Planning (OBP), Knowledge-Based Planning (KBP)[1][2] and so on, which can support the solution of large problems and improve the decision ability effectively.

\section{Capability Model}

The capability of simulation entity depends on resources it has, and affects the result of the task processing. The result usually depends on abstract field features. The result can depend on some single index, such as finish time, effectiveness and consumed resources. It can depend on comprehensive assessment of indexes, such as the weighted average of time, effectiveness and consumed resources. Therefore the capability has the field feature at some degree.

Simulation plans in different operation mode are different, each plan should include some key points. For example, in the task of destroying objects, simulation plan should include four aspects as follows: way of destroying, effectiveness of destroying cost of destroying and action constraint. Once it meets the above conditions even that it hasn't carried out such task, the simulation entity can be considered to have the ability to simulate the task of destroying objects. So the capability-based task analysis is one method independent of field.

The premise of using capability to do task analysis is to build capability model, which at least includes a task set, state space and domain description. The domain description of capability model in figure 1 mainly includes such factors like the weather, terrain and resources. The state space means the prior and subsequent task set. And the task set includes the operation means of attacking or 
defensing that the model has. Those above elements can all be described with the conceptual model of task.

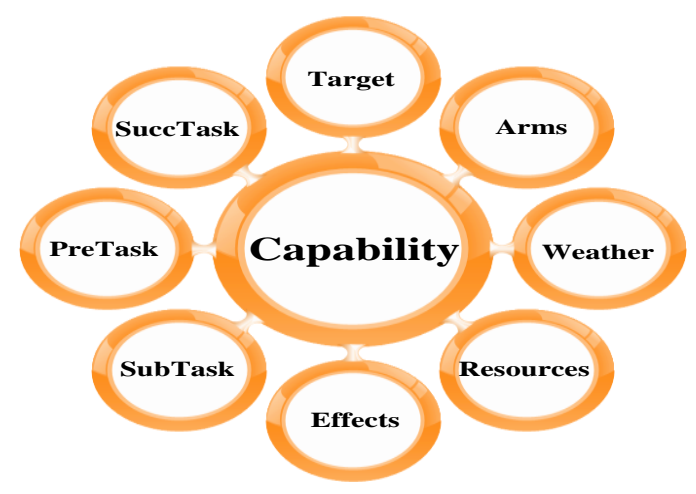

Fig. 1 Capability Model

\section{The simulation combination analysis based on capability model}

As each simulation task depends on the target, each task has at least one target, which means that each entity has the capability to accomplish this task. Therefore, with the task model, we can get the capability requirement target by analyzing task. Then with the objective, necessary simulation nodes, simulation activities and resources can be got to achieve this objective. Objective analysis is a process of decomposing step by step. And the result of it is to form a task capability tree. The decomposition mode of capability objective takes the Achieve Mode[3] of KAOS[4] into consideration and finally forms the AND/OR tree. The decomposition of task is AND decomposition. If a task has different kinds of operation means or capabilities, OR decomposition plays a role. The process of decomposing is shown in figure 2. In the figure, field objective model is a common decomposition method provided by field experts. This model can be detailed when application requirements are achieved. That is to say that field objective can be divided into many application objectives. However, when it is to decompose the field objective of leaf node, artificial decomposition may be needed.

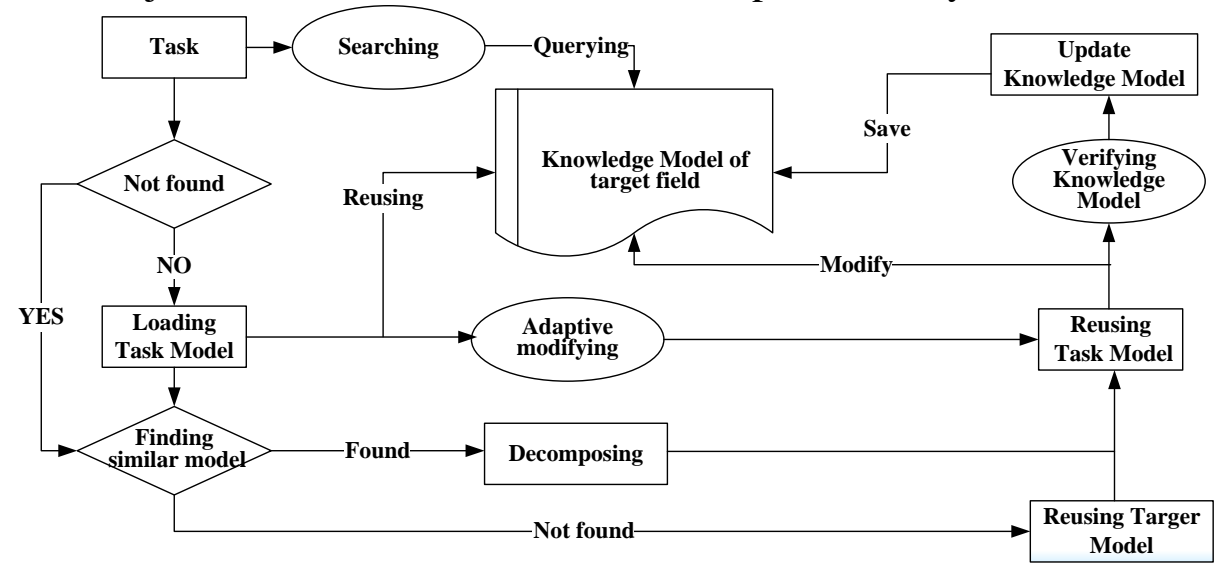

Fig. 2 decomposing based on capability

\section{A Simulation Combination Algorithm Based on CBR and HTN}

In the process of social and military practice, people accumulate a lot of valuable cases [5][6]. If these cases can be made full use of, the efficiency of simulation system will be improved largely. With combining the simulation task and the retrieval process of these cases, the experience can be used for reference. Then the task planning time can be decreased. Finally the efficiency and precision of operation planning can be improved.

Considering the above, s stands for the state. Target stands for planning objective. $\mathrm{C}$ stands for the constraint set. $\mathrm{O}$ stands for the operation set. Then the planning algorithm based on CBR and HTN can be described as follows: 
CBR_HTN(s,Target,C,O)

(1) If (Target, C) can be got, turn to step 2. If not, turn to step 6.

(2) If the decomposition of capability model exists to meet the Target requirement, turn to step 3. If not, turn to step 4.

(3) Retrieve and turn back the case result that meets the constraint conditions. Then turn to step 7.

(4) Considering the Target requirement, build the capability model and form objective task capability set. Then turn to step 6 .

(5) Ask if assistance is needed and publish the Target requirement in the planning network. If the feedback can be got during the waiting period, turn to step 1 . If not, build capability model manually and ascertain the objective task capability set $T$, finally turn to step 6 .

(6) If $T \neq \varnothing$, turn to step 6 . If not, for $\mathrm{i}=1$ to Count (T) and execute the following:

a) If CBR_HTN(s, Ti, C, O) doesn't have some similar case result, turn to step b. If not, turn step e.

b) Ask interactively that if assistance is needed. If it is needed, turn to step c. If not, turn to step d.

c) Retrieve the similar case result of $\mathrm{Ti}$ in the network. If the result exists, turn to step e. If not, turn to step d.

d) Edit the plan of executing the task manually.

e) Ascertain and turn back the locally optimal solution.

(7) Edit and adjust the task. Then finish the planning.

During the whole planning process, the core is task to decomposition and case retrieving. After decomposing the task each time, retrieve the local case library and network case library to check if there are some similar cases. If not, continue decomposing or adjust manually. If there are some similar cases, get and modify the case data. In practice, case will adjust according to the specific situation. Therefore the case knowledge library $\mathrm{K}$ of planning is composed of incomplete field description I and case library B. That is $\mathrm{K}=\mathrm{I} \cup \mathrm{B}$. Moreover, in the planning process, the case offers two kinds of knowledge. One is the knowledge about task decomposition. Because the case is about the algorithm, use the case just like the replay of some planning. The other one is knowledge about field.

\section{Analysis of Example}

An example about how to form the simulation plan of destroying a bridge is shown to illustrate the planning algorithm. This task is a typical application with many implementation methods, such as aerial bombardment, ground assault and precise strike. In the operation, different methods have different key points. For example, aerial bombardment will make sure whether the enemy sets out air force or not, whether to deploy the air defense and the weather. And ground assault will make sure the line of attack, whether the enemy deploys the ground forces in our heading direction, and whether to need the air cover when the army drives in.

What the commander needs to consider is that how to complete the task. First, commander should describe the operation intention to the simulation system. Then analyze the task and form the plan. Finally give the commands. In the process, the planning part is to get a serial of task quickly according to commanders' intention so that the more detailed plan can be got.

According to the relevant research, the task analysis method based on capability model can be used to make sure the operation intention. And the intention is simply described as follows:

Tab. 1 description of planning task

\begin{tabular}{|l|l|}
\hline task & destroying great bridge A \\
\hline deadline & $2052-01-2512: 30: 30$ \\
\hline goal & Heavy armored vehicles can't pass \\
\hline
\end{tabular}

Planning process:

Step 1: Build the top capability model interactively. With the help of interactive inquiry, build the model quickly which can meet the requirement of top capability so that planning staff can develop in the next period. Figure 3 shows that common attack methods include the air attack and ground assault. 
And the air attack can be divided into attacks from air force and Second Artillery Corps. In practice, commander can adopt more flexible operation mode to analyze the situation clearly.

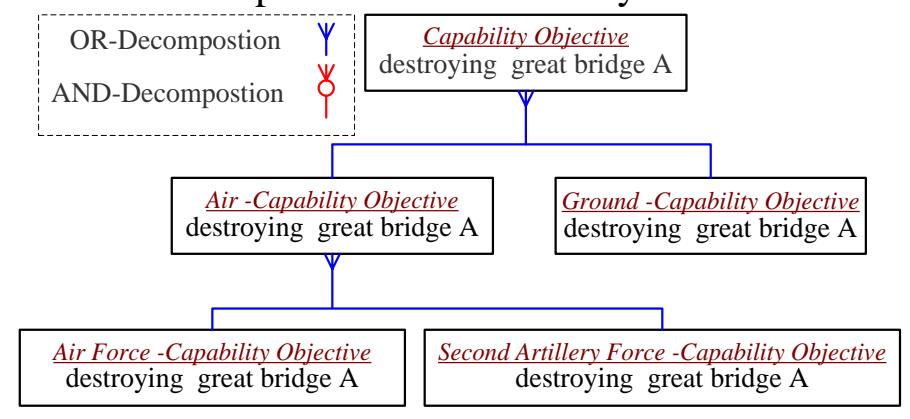

Fig. 3 top-level Capability Model of destroying great bridge A

Supposing that command think air-ground cooperative combat is feasible, the capability model will be adjusted as Fig 4.

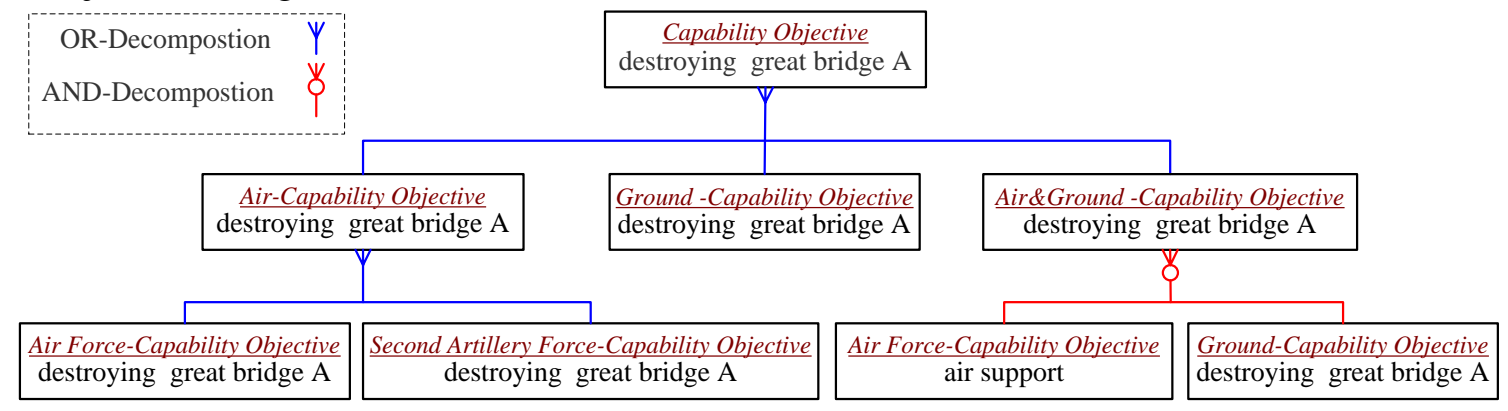

Fig. 4 extended Capability Model of destroying great bridge A

Step 2: Make the task planning of capability objective. With step 1, we can know that there are four operation modes of destroying bridge A, ground assault, air attack, precise attack and air-ground cooperative combat. According to capability model, the characteristic attribute of objective task can be described as follows:

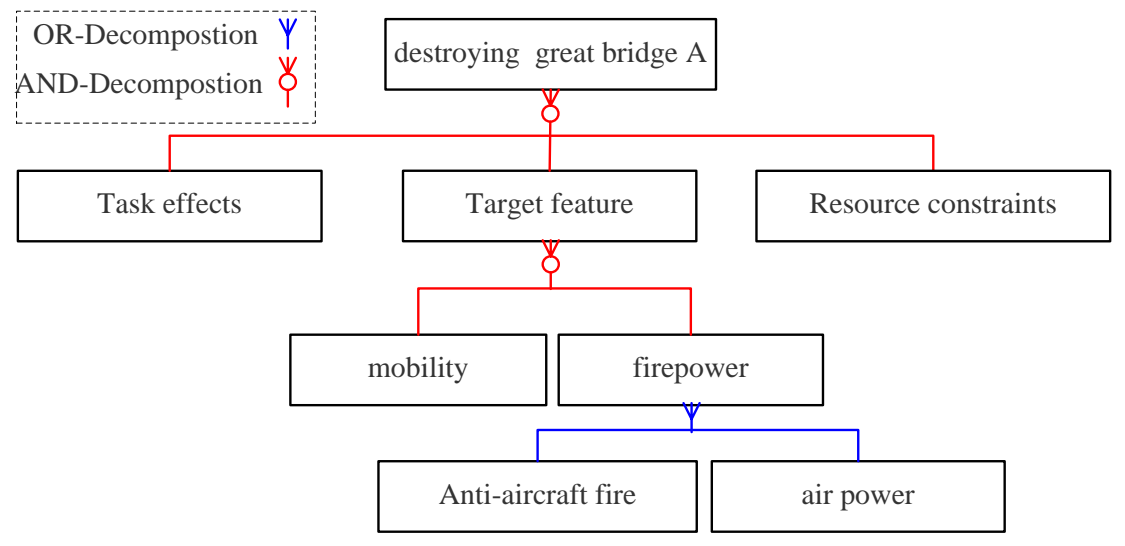

Fig. 5 characteristic properties of destroying great bridge A

From the figure 5, we can know that attacking the ground objective should take mobility, anti-air capability, and air support capability into consideration. Therefore, the sub-task of top level can be decomposed as follows:

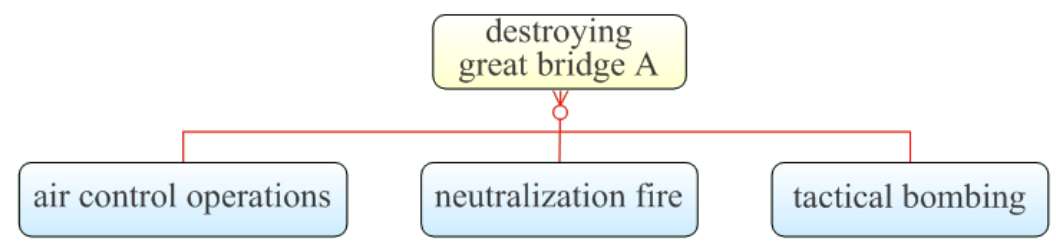

Fig. 6 Task sets of destroying great bridge A 
Step 3: Retrieve the similar case of the three tasks, i.e. "air fighting", "fire suppression" and "bombing targets", from the local and network case library. Suppose "air fighting" and "fire suppression" have found the similar cases, and "bombing targets" haven't. At this moment, add cases of "air fighting" and "fire suppression" into planned task. Suppose the 22nd Bombing Division 33rd Regiment can meet the capability requirements. Therefore complete the whole analyzing process, and get the whole plan described in the following figure.

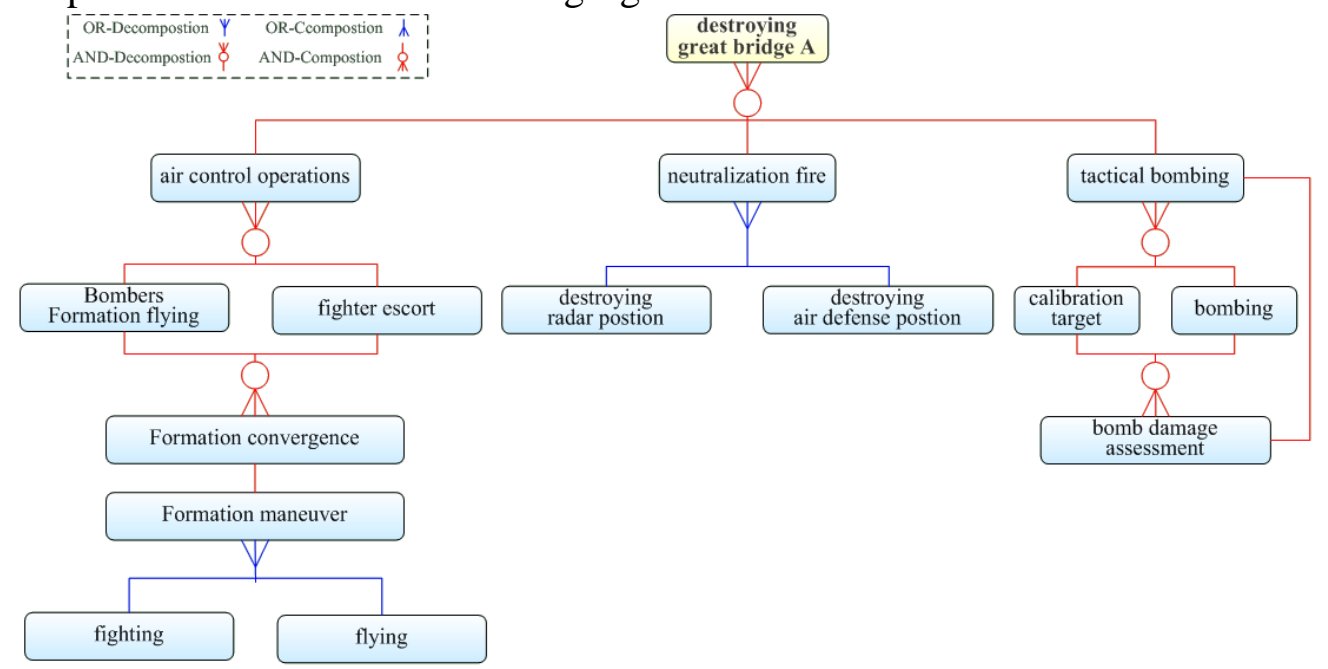

Fig. 7 task decomposing of destroying great bridge A

\section{Summary}

The dynamic combination technique in simulation application has lots of advantages, which make it be the most important method to integrate the simulation application in the future environment of Global Information Grid. And how to improve the dynamic combination efficiency and expand the combination is the key question. So based on capability requirement, first, we analyze the combination process of capability-oriented application. And then we propose a Simulation Combination Algorithm. Finally an example is illustrated to verify the effectiveness of this algorithm, which provides an important thought for dynamic and real-time simulation of operations. Moreover, the next job is to do some research on integrated techniques of historical simulation system.

\section{References}

[1] Luo Jiewen, Shi zhiping, Heqing, et al. A Quick Emergency Response Plan Generation System Combining CBR and RBR. Journal of Computer Research and Development:2007,44(4): 660-666.

[2] YANG Lifeng, KOU Ziming. Research on Rapid Generation Technique for Environment Emergency Plan. Journal of TAIYUAN UNIVERSITY OF TECHNOLOGY:2013, 44(5): 612-614.

[3] LEVCHUK G, GRANDE D, PATTIPATI K, et al. Mission Plan Recognition: Developing Smart Automated Opposing Forces for Battlefield Simulations and Intelligence Analyses. 13th CCRTS, 2008.

[4] WU Xiao, CHONG Yu-Zhen, NI Hong-bo, WANG Hai-peng. Smart home inference system combining CBR and RBR. Application Research of Computers:2009,26(3): 977-979.

[5] CHEN Ai-Xiang, JIANG Yun-Fei, CHAI Xiao-Long et al. Structure-Oriented Learning-Based Planning Method. Journal Software:2014,25(8): 1743-1760.

[6] LIU Yong, LUO Chen, QUAN Ji-chuan et al. Collaborative Planning of ECP Basing on Multi-Agent.2th CICC,2014. 